\title{
LIX. The collisions of electrons with molecules of a gas
}

\section{J.S. Townsend F.R.S.}

To cite this article:J.S. Townsend F.R.S. (1920) LIX. The collisions of electrons with molecules of a gas , Philosophical Magazine Series 6, 40:238, 505-511, DOI: 10.1080/14786441008636154

To link to this article: http://dx.doi.org/10.1080/14786441008636154

曲 Published online: 08 Apr 2009.

Submit your article to this journal $ऍ$

Џ Article views: 8

Q View related articles $\square$

Citing articles: 2 View citing articles 5 
LIX. The Collisions of Electrons with Molecules of a Gas. By J. S. Townsend, F.R.S,. Wykeham Professor of Pliysics, Oxford*.

$\mathbf{I}^{\mathrm{N}}$ a paper on Ionization by Collision P. O. Pedersen $\dagger$ gives a method of calculating ionization potentials from the currents obtained through a gas between parallel plates at various distances apart, when electrons are set free from the negative electrode. In these calculations it is assumed that each collision with a molecule of air has the effect of reducing the velocity of the electron to zero, and the following formula is obtained by Pedersen for the total number $n_{a}$ of electrons arriving at the positive electrode when $n_{0}$ electrons start from the negative electrode,

$$
n_{a}=n_{0} e^{\gamma \alpha\left(a-l_{0}\right)},
$$

the additional ions being generated by collisions in which the velocity of the electrons exceeds a certain critical value.

This formula is applied to the following numbers, which were published several years ago as an example of the experiments on which the theory of ionization by collision was founded $\ddagger, a$ being the distance between the plates in centimetres, and $q$ the currents in arbitrary units. The experiments were some of those made with air at a pressure of one millimetre, when electrons are set free from the negative electrode by the action of ultra-violet light, the force being 350 volts per centimetre:-

$\begin{array}{ccccccc}a \ldots \ldots \ldots \ldots . & \cdot 2 & \cdot 4 & \cdot 6 & \cdot 8 & 1 \cdot 0 & 1 \cdot 1 \\ q \ldots \ldots \ldots \ldots & 2 \cdot 86 & 8 \cdot 3 & 24 \cdot 2 & 81 & 373 & 2250\end{array}$

The shortest distance between the plates at which a measurement of current was made was $\cdot 2$ centimetre, as it was necessary to allow the electrons starting from the negative electrode to traverse a certain distance in the gas in order that they should attain the final average velocity corresponding to the electric force. The currents at the shorter distances $\cdot 2, \cdot 4$, and $\cdot 6$ centimetre between the plates are in geometrical proportion, and may be represented by the simple formula $n_{a}=n_{0} \epsilon^{\alpha a}$ given at the time the experiments were first published, and the only quantity which can be deduced from the ratio of these currents is $\alpha$ the coefficient

* Communicated by the Author.

† P. O. Pedersen, Phil. Mag. July 1920, p. 129.

† J. S. Towusend, Phil. Mag. (6) vol. vi. p. 598, Nov. 1903.

Phil. Mag. S. 6. Vol. 40. No. 238. Oet. 1920. 2 L 
of $a$ in the exponential term. Admitting that the ionization due to positive ions produces appreciable effects at the larger distances, the currents at the distances $\cdot 8,1 \cdot 0$, and $1 \cdot 1$ centimetre between the plates cannot be represented by a formula of this type.

Thus in the notation adopted by Pedersen the only grantity which can be determined directly from these experiments is the product $\gamma \alpha$, and by means of a theoretical investigation the ionizing potential in volts is found to be 16 . But it appears from other experiments that no reliable estimate of the velocity of an electron required to ionize a molecule can be obtained from the value of $\gamma \alpha$ by a calculation founded on the supposition that a large proportion of the kinetic energy of an electron is lost at each collision. I have already pointed this out in various publications, but it may be of interest to refer to a series of experiments which show how the velocities of electrons are distributed when moving under an electric force in a gas, and afford a more reliable means of estimating the velocities required to generate ions by collisions.

2. In the first * of this series of investigations of the motion of electrons in gases, experiments were made on the diffusion of electrons moving in air under forces much smaller than those required to generate ions by collisions. It was found that in an electric field the electrons moved freely and acquired a mean velocity of agitation which is much larger than the normal value corresponding to thermal equilibrium with the molecules of the gas. This shows that when an electron collides with a molecule of air it retains to a remarkable extent a large proportion of the kinetic energy acquired under the electric force. A similar result was obtained in oxygen, hydrogen, and carbonic acid. For a given force $Z$, the maximum pressure of the gas at which the electrons move freely, varies in different gases; this pressure is greater in hydrogen than in air, and in carbonic acid it is less than in air. Subsequently Franck and Hertz $\dagger$ found by another method that electrons after colliding with molecules of helium or hydrogen retained a large proportion of the energy acquired under an electric force, and the collisions were described as being of an elastic type. 'Reference has been made so frequently to this result that the phenomenon is generally supposed to be peculiar to monatomic gases or hydrogen.

* J. S. Townsend, Proc. Roy. Soc. lxxxi. p. 464 (1908). 1913.

† J. Franck \& G. Hertz, Verh. d. D. Phys. Ges. xv. 9. p. 373, May 
3. When considering the velocities of electrons in air under an electric force, both the motion of agitation and the motion in the direction of the electric force must be taken into consideration; for even when the forces are sufficiently large to produce appreciable numbers of additional electrons by collisions, the inean velocity of agitation may be greater than the mean velocity in the direction of the electric force. Let $\mathrm{IV}$ be the mean final velocity of a stream of electrons in the direction of the electric force $\mathrm{Z}$ in a gas at pressure $p$, $u$ the mean final velocity of agitation. The velocities $W$ and $u$ have been found for air over large ranges of forces and pressures, and the values ohtained are in good agreement with the general kinetic theory. 'Thus the quantities $W$ and thave been found to depend on the ratio $Z / p$, and since $"$ increases with the force the velocity $W$ is not proportional to the force. The experiments are comparatively simple as they only involve the determination of the distribution of electrons in a stream moving under an electric force, and the deflexion of the stream by a magnetic force of small intensity. If the motion of agitation were in thermal equilibrium with the molecules of the gas the lateral diffusion of the stream of electrons would tollow certain simple laws, but in practice the lateral diffusion is found to be abnormally large owing to the increased velocity of agitation, and the factor $k$ by which the energy of agitation exceeds the normal value corresponding to thermal equilibrium is easily obtained from the experiments. The normal mean velocity of agitation $u_{0}$ of electrons in equilibrium with molecules of a gas at $15^{\circ} \mathrm{C}$. is approximately $1 \cdot 14 \times 10^{\top} \mathrm{cm}$. per second, so that the acturil mean velocity of agitation when the electrons are moving in an electric field is $1.14 \times 10^{7} \times \sqrt{ } k$. The following table * gives the values of $\mathrm{W} \times 10^{-6}$ and $u \times 10^{-6} / 1 \cdot 14=10 \sqrt{k}$ for electrons moving in dry air, obtained from experiments in which the forces ranged from 2 to 20 volts per contimetre, and the pressures from $\cdot 25$ to 185 millimetres.

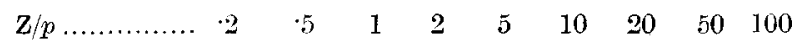

$$
\begin{aligned}
& \begin{array}{lllllllllll}
\mathrm{W} \times 10^{-6} & \ldots \ldots & \cdot 5 & 9 & 1.25 & 1.75 & 3.0 & 5.2 & 9 \cdot 0 & 173 & 27
\end{array} \\
& \begin{array}{lllllllllll}
\frac{u \times 10^{-b}}{1.14} & \ldots \ldots & 16 & 24 & 34 & 47 & 62 & 67 & 75 & 101 & 127
\end{array}
\end{aligned}
$$

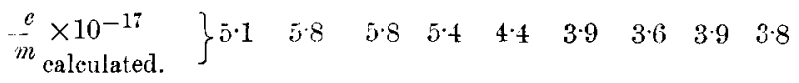

* J. S. Townsend \& H. T. Tizard, Proc. Roy. Soc. Ixxxviii. p. 336 (1913). 
4. In order to draw conciusions from these results by representing the motion of the electrons as taking place in a series of free paths beiween collisions with molecules, it is necessary to specify the distance between an electron and the centre of a molecule when a collision may be considered to take place. This distance most probably depends on the velocity of the electron, but with the velocities which are here considered, the values of $k$ and $W$ as found experimentally are in fair agreement with calculations made on the bypothesis that collisions occur with molecules when the distance between the electron and the centre of a molecule is equal to the diameter of the molecule as determined in the kinetic theory of gases. This is seen by means of the formula given by Langevin * for the velocity $\mathrm{IV}$ in terms of molecular quantities. In the case of electrons where the mass $m$ of an electron is small compared with the miss of a molecule of the gas the formula becomes

$$
\mathrm{W}=\frac{\mathrm{Z} e}{m} \times \frac{l}{u} \times \cdot 815,
$$

$l$ being the mean free path, and $u=u_{0} \sqrt{k}$ the velocity of agitation of the electron. 'The formula may be applied to test the numbers given in the above table for the smaller values of $\mathrm{Z} / p$ where $u$ is large compared with $W$. The mean free path $l$ is aken as $1 / \pi \sigma^{2} v$, where $\sigma$ is the radius of a molecule of air, $\nu$ the number of molecules per cubic centimetre at a millimetre pressure. The value of $l$ thus obtained is $3 \cdot 2 \times 10^{-2} \mathrm{~cm}$.

When the above equation is written in the form

$$
\mathrm{W}^{2}=\frac{\mathrm{Z}^{2} e}{m} \times \frac{v e}{m v u_{0}{ }^{2} k} \times l^{2} \times \cdot 664,
$$

and the known values of $v e$ and $m \nu u_{0}{ }^{2}$ are substituted, it reduces to

$$
\frac{e}{m}=\left[\frac{W p}{\mathrm{Z}}\right]^{2} \times \frac{k}{2 \cdot 8}
$$

so that the ratio of $e / m$ may be calculated from the values of $\mathrm{W}$ and $k$. The values of $e / m \times 10^{-17}$ thus obtained are given in the fourth row of the above table, and those corresponding to the smaller forces are in good agreement with the known value $e / m \times 10^{-17}=5 \cdot 3$. This result confirms the conclusion already arrived at as to the free movement of the electrons.

5. With forces from 50 to 100 volts per centimetre, the number of ions generated by collisions with molecules of air

* P. Langevin, Ann. de Clim. et de Phys. (8) v. p. 245 (1905). 
at a millimetre pressure will depend on the distribution of the velocity of agitation, which is much greater than the velocity in the direction of the electric force. When an electron travels a distance of one centimetre in the direction of the electric force the total length of its trajectory is $v / W$ approximately, and the total number of collisions it makes with molecules is $u / \mathrm{Wl}$, where $l$ is the mean free path of the electron. If $\alpha$ be the number of molecules ionized by an electron in moving a distance of one centimetre in the direction of the force, the ratio of the number of collisions in which ionization takes place to the total number of collisions is $(\alpha W l) / u$.

In order to fins an a verage vilue of the velocity $\mathrm{V}^{\prime}$ of the electron required to ionize a molecule the quantity $(\alpha \mathrm{W} l) / u$ may be equated to the ratio of the number of collisions in which the velocity exceeds $V^{\prime}$ to the total number of collisions. Assuming the velocities of the electrons to be distribuied according to Maxwell's law it is easy to show* that the latter ratio is

$$
\epsilon^{-\frac{3 V^{\prime 2}}{2 k u_{0} u^{2}}}\left(1+\frac{3 V^{\prime 2}}{2 k u_{0}^{2}}\right)
$$

It is convenient to represent the velocity $\mathrm{V}^{\prime}$ in terms of the potential $\mathrm{P}$ in volts required to give this velocity to a particle with an atomic charge. The value of $P$ in terms of $V^{\prime}$ is given by the relation $\frac{V^{\prime 2}}{u_{0}^{2}}=27 \times \mathrm{P}$, and the equation to
determine $\mathrm{P}$ becomes

$$
\frac{\alpha \mathrm{Wl}}{u}=\epsilon^{-\frac{81 \times \mathrm{P}}{2 k}}\left(1+\frac{81 \times \mathrm{P}}{2 k}\right) .
$$

The values of $P$ thus ohtained are $23 \cdot 7,26,28,29$ volts from the experiments with the smaller forces in which the values of $Z / p$ were $40,50,70,90$ respectively.

It might seem that this method of finding the potential $P$ would be inaccurate, as the errors of several experimental determinations would be involved. But the values of $\mathrm{P}$ as given by the above formula are only slightly affected by large errors in the values of $\alpha, l$, and $W$, and the quantity $k$ is the only factor which need be known accurately. 'l'he principal error in the calculation is probably due to talking Maxwell's law as giving the distribution of the velocities of electrons acted on by an electric force. It should be remembered that the potentials $P$ thus found are average values, and with the definition of a collision that has

* Phil. Mag. (6) vol. xxrii. p. 269 (1914). 
been adopted it is most improbable that ionization takes place in all collisions in which the veiocity exceeds the value correspondin to the voltage $\mathrm{P}$, or that ionization does not take place in a collision in which the velocily is less than the value.

The potentials $\mathrm{P}$ do not therefore represent a critical potential, and the minimum potential required to ionize a molecule when the collision occurs under the most favourable conditions should be less than the above values. In air, the minimum ionizing potential as found by Lenard* by a different method is 11 volts; but it may be concluded that ionization is produced in a comparatively small number of cases when electrons collide with a velocity corresponding to this roltage. In air at one millimetre pressure the observed increase of conductivity due to the motion of electrons would be accounted for if ionization took place in about 2 per cent. of all the collisions in which the velocity was greater than that corresponding to 11 volts, when $Z$ is 100 volts per centimetre, and in about 4 per cent of these collisions when $\mathrm{Z}$ is 50 volts per centimetre.

6. The numbers obtained for the velocities $W$ and the factor $k$ provide a simple means of measuring the proportion of the energy of an electron which is lost on colliding with a molecule. Thus with air at a pressure of one millimetre, and a force of 20 volts per centimetre, the value of $k$ is $56 \cdot 3$, so that the mean final velocity of agitation corresponds to a potential of $2 \cdot 1$ volts, and in passing through a distance of one centimetre in the directicn of the force the electron makes 290 collisions with molecules. Thus in the final steady state of motion the energy corresponding to a potential fall of 20 volts is dissipated in 290 collisions, so that the average loss of energy at ench collision is $1 / 14 \cdot 5$ volt. Thus about 3 per cent. of the energy is lost on each collision, or about 1.5 per cent. of the velocity, when collisions take place with velocities of $8.5 \times 10^{7}$ centimetre per second.

The exact value of the elasticity has been found by Pidduck + taking into consideration the distribution of the velocities. If the collisions are perfectly elastic the following relation is obtained between the values of $\mathrm{W}$ and $k$ :

$$
k-1=\frac{W^{2}}{\Omega^{2}},
$$

$\Omega$ being the mean velocity of agitation of the molecules of the gas.

* P. Lenard, Ann. der Phys. (4) viii. p. 194 (1902).

† F. B. Pidduck, Proc. Roy. Soc. A. Ixxxviii. p. 296 (1913). 
On ihe Pressure on the Poles of an Electric Arc. 511

If $\epsilon$ be the coefficient of restitution, and $f=\frac{1}{2}(1+\epsilon), f$ is unity when the collisions are perfectly elastic, but a very small reduction in $f$ below this value has the effect of making a large reduction in the velocity of agitation. The followin" are the values of $f$ found by Pidduck corresponding to the observed value of $k:-$

$\begin{array}{ccccc}Z / p \ldots \ldots \ldots \ldots \ldots . & \cdot 2 & 2 & 20 & 150 \\ k-1 \text { observed... } & 1.8 & 20 & 55 & 210 \\ f \ldots \ldots \ldots \ldots \ldots \ldots . & .9993 & .9988 & .99 & .96\end{array}$

Thus as the velocity of the electrons increases, the elasticity tends to diminish, but with the smaller velocities the molecules may be considered to be perfectly elastic.

LX. On the Pressure on the Poles of an Electric Arc.

To the Editors of the Philosophical Magazine.

Gentlemen,-

I his latest publication under the above title Prof. Duffield 1 (Proc. Roy. Soc. June 1920), discussing his results, tries to show that the motion of ions within an electric arc and the electric wind could not be expected to produce a pressure upon the poles of the arc. Having myself contributed to your Magazine a few papers on the electric wind, will you once more give me your hospitality in order to clear up some points in connexion with this question?

I an very much indebted to Sir. J. J. Thomson for having shown to me some time ago (in a way similar to that used by Prof. Duffield) that ions moving under an electric force towards an electrode (evidently of opposite sign) cannot produce a pressure upon it, the pull on this electrode during the motion of the ions being balanced by the impact of the ions on the electrode. Prot. Duffield, however, has entirely overlooked the fact that ions moving from an electrode (of the same sign) produce a reaction upon this electrode which is not compensated by any other force. Consequently, a strong pressure upon the poles of an electric arc is to be expected as a result of the motion of the ions within the arc. Moreover, when the experiments are carried out not in a vacuum (as in the case of Prof. Duffield's experiments), the energy of the ions is partly transferred by collision to the neutral gaseous molecules, which results in the formation of a stream of the surrounding atmosphere in the direction of the electric field -a phenomenon known as the electric wind. Experiments show that only a small part of the momentum of this stream 